\title{
Chiral Metal as a Heisenberg Ferromagnet
}

\author{
H. Mathur ${ }^{1,2}$ \\ ${ }^{1}$ Department of Physics, Case Western Reserve University, Cleveland, Ohio 44106-7079 \\ ${ }^{2}$ Institute for Theoretical Physics, University of California at Santa Barbara, Santa Barbara, CA 93106-4030
}

(February 5, 2020)

\begin{abstract}
The two-dimensional surface of an integer quantum hall multilayer is mapped onto a Heisenberg spin-chain with ferromagnetic coupling. Using this mapping it is shown non-perturbatively that the surface states constitute a very anisotropic metal in the infinite size limit. For multilayers of finite size, two diffusive mesoscopic regimes are identified and the conductance fluctuations are calculated perturbatively for both. The Heisenberg spin-chain representation is used to study the directed wave problem and the exact result is obtained that the mean-square deflection of a directed wave grows as the square root of the propagation distance.
\end{abstract}

PACS: 72.15.Rn; 75.10.Jm; 73.40.Hm; 42.25.Bs

Dirty electronic systems exhibit a variety of phases many of which are not well-understood [1], [2]. For example electrons moving in two dimensions under the influence of a magnetic field exhibit a sequence of localizationdelocalization transitions as the magnetic field or some other system parameter is varied. Understanding of these transitions, which underlie the quantum hall effect, is based largely on numerical simulation [3].

The purpose of this Letter is to study the two dimensional electronic states that live on the surface of an integer quantum hall multilayer (sometimes called the bulk quantum hall effect). At the edge of each quantum hall layer the electrons circulate in one sense only and may be modelled as non-interacting chiral fermions 㭡. If the layers are coupled by tunneling, the surface states comprise a two-dimensional chiral electronic system (see fig 1). The Bechgaard salts are a natural realization [5] and it is also possible to fabricate an appropriate semiconductor heterostructure [6]. It will be seen below that the chiral model used to describe the surface states of the quantum hall multilayer (eq 1 below) bears some similarity to a model for the quantum hall effect introduced by Chalker and Coddington [7]. However there is a crucial difference which makes the chiral model tractable whereas the Chalker-Coddington model has defied solution.

The key question from the point of view of quantum transport is whether the surface electronic wave functions are localized or extended along the direction of the field (the $z$-direction in fig 1 ). This determines whether the system is metallic or insulating since transport along the $z$-direction would be dominated by the surface. In either case, to fully characterize the transport it is not sufficient to study the disorder-averaged conductance: Localized electronic systems generally possess a very broad distribution of conductances. Although in contrast metallic systems of ordinary (rather than chiral) electrons do not have a broad conductance distribution, the conductance fluctuations of finite-sized or mesoscopic metallic grains have remarkable universal properties [8] (for example, the typical fluctuations are of order $e^{2} / h$-independent of the mean conductance or other details of the sample).

Quantum transport in the chiral model has previously been studied numerically [9] and by mapping onto field theories 10 12]. In particular Balents et al. 12] have mapped the system onto a one-dimensional supersymmetric ferromagnetic spin-chain using the wellestablished supersymmetric technique for performing disorder averages [13]. Using this mapping they are able to establish the important result that the two-dimensional chiral model is metallic (in the sense that the conductance scales ohmically) even for arbitrarily strong disorder. This should be contrasted with conventional electronic systems in which metallic scaling is associated with large conductance and which are generically localized in two dimensions [1]. The surface states of the bulk quantum hall system are thus revealed to be a novel metallic phase with interesting localization and mesoscopic fluctuation physics that awaits exploration.

The chiral model (eq 1 below) that describes the surface of a quantum hall multilayer is of interest from another quite distinct point of view. It should apply whenever waves propagate in a medium that is sufficiently anisotropic to warrant neglect of backscattering in one direction. The problem of waves propagating in an anisotropic medium has been the focus of much attention and is known as the directed wave problem (see for example ref 14, 15, and references therein). Although directed waves are described by the chiral model the question one asks in this context is very different: The electron is assumed to be localized at a single point initially. Thereafter it moves ballistically in the chiral $x$-direction and the wave function spreads (presumably diffusively) in the transverse $z$-direction. The interesting questions here concern the growth of the wave packet width and the fluctuations in the position of the centre of the wave- 
packet (denoted $\left[<n>^{2}\right]_{\text {imp }}$; the notation is explained below). The broadening of the width can be easily calculated and the answer has been known since the 1970s [16]. $\left[\langle n\rangle^{2}\right]_{\mathrm{imp}}$ is more difficult to calculate as the average of four green's functions is now required; it has not been evaluated within this model previously.

In this paper a new approach to disorder averaging is introduced which is distinct from the conventional replica or supersymmetry methods and is especially adapted to this system. Using this method it is possible to map the chiral model (in the limit of infinite size) onto a much simpler soluble one-dimensional model: an ordinary Heisenberg ferromagnet. The absence of localization for the chiral system established by ref [9, 10,12 then emerges non-perturbatively as a consequence of the well-known quadratic dispersion of ferromagnetic magnons. The advantages of this mapping onto an ordinary ferromagnet become evident when a more difficult calculation is attempted. For example $\left.[<n\rangle^{2}\right]_{\text {imp }}$ can be expressed in terms of the matrix elements of two-magnon states of the ferromagnetic representation. Although magnons interact, it is not difficult to obtain the two-magnon eigenstates of a ferromagnet [17]. Carrying out such a calculation leads to the exact result that $\left[\langle n\rangle^{2}\right]_{\text {imp }}$ grows as $x^{1 / 2}$. This exact result agrees with the numerical simulations of 18 but contradicts those of [19]; it also agrees with results obtained from a simplified lattice model of directed wave propagation introduced by Saul, Kardar and Read [15,20,21]. In their model special assumptions are made about the disorder which make it possible to directly evolve the probability function (the modulus square of the wave function) without reference to the wave function itself. The results of this paper show that the same behaviour results for a more generic disorder distribution.

Finally the properties of mesoscopic quantum hall multilayers are studied. Due to the anisotropy of the surface states two diffusive mesoscopic regimes can be identified. Following the terminology of ref 12 in the onedimensional diffusive regime electrons are typically able to wind around the sample in the chiral direction many times before diffusing across in the $z$-direction. The opposite limit of sufficiently large circumference that winding paths are rare is called the zero-dimensional regime. The mesoscopic regimes are difficult to analyse due to the winding paths. Spectral correlations were computed in ref 12] in the zero-dimensional limit; however spectral correlations are difficult to probe experimentally. Here conductance fluctuations are calculated in the two mesoscopic regimes using the standard methods of impurity averaged perturbation theory [8]. Universal conductance fluctuations of order $e^{2} / h$ are found in the onedimensional limit coinciding with the result for ordinary metals. Interestingly, there is a crossover to much larger fluctuations in the zero-dimensional diffusive limit.

The precise model used for the multilayer surface is now described. Each quantum hall layer has as many edge channels as there are filled Landau levels. For simplicity we shall focus on the case of just one filled Landau level. The surface of the multilayer is then a two dimensional chiral electronic system governed by the Schrödinger equation

$$
\begin{array}{r}
\left(-i v \frac{\partial}{\partial x}+V_{n}(x)-E\right) G_{E}^{R}\left(n, x ; n^{\prime}, x^{\prime}\right) \\
-t\left\{G_{E}^{R}\left(n+1, x ; n^{\prime}, x^{\prime}\right)+G_{E}^{R}\left(n-1, x ; n^{\prime}, x^{\prime}\right)\right\} \\
=-i v \delta_{n, n^{\prime}} \delta\left(x-x^{\prime}\right) .
\end{array}
$$

Here $G_{E}^{R}$ is the retarded Green's function at frequency $E$. $t$ produces interlayer hopping and $V_{n}$ is the disorder potential. Units are chosen so that the edge velocity $v=1$, the interlayer separation $a=1$ and $\hbar=1$. The anisotropy and chiral character of the model are reflected by the fact that the equation is first order in the chiral $x$ - direction whereas it is second order in the transverse direction.

A mapping onto a one-dimensional problem is obtained by noting that in the limit of infinite size (but in that limit only) the circumference $C \rightarrow \infty$ and the retarded Green's function obeys the chiral boundary condition $G_{E}^{R}\left(n, x ; n^{\prime}, x^{\prime}\right)=0$ for $x<x^{\prime}$. Due to this boundary condition, it is possible to interpret eq (1) as the timedependent Schrödinger equation for a one-dimensional tight-binding model with the chiral co-ordinate $x$ identified as time and $G_{E}^{R}\left(n, x, ; n^{\prime}, x^{\prime}\right)$ identified as the timedomain retarded Green's function. Note that the onsite energies of the tight-binding model fluctuate in time. It is convenient to make a gauge transformation $G^{R} \rightarrow$ $G^{R} \exp \left[i \gamma_{n}(x)-i \gamma_{n^{\prime}}\left(x^{\prime}\right)\right]$ where $\partial \gamma_{n}(x) / \partial x=V_{n}(x)-E$. In this gauge eq (1) becomes

$$
\begin{aligned}
& -i \frac{\partial}{\partial x} G_{E}^{R}\left(n, x ; n^{\prime}, x^{\prime}\right)-t_{n}(x) G_{E}^{R}\left(n+1, x ; n^{\prime}, x^{\prime}\right) \\
& -t_{n-1}^{*}(x) G_{E}^{R}\left(n-1, x ; n^{\prime}, x^{\prime}\right)=-i \delta_{n, n^{\prime}} \delta\left(x-x^{\prime}\right) .
\end{aligned}
$$

Here $t_{n}(x) \equiv t \exp i\left[\gamma_{n+1}(x)-\gamma_{n}(x)\right]$. The random potential is thus eliminated but the hopping matrix elements are now modulated by random phases.

The tight-binding model can be rewritten in secondquantized language by introducing $c_{n}^{R \dagger}$ and $c_{n}^{R}$ which create and annihilate fermions on site $n$ of the tight-binding lattice and which evolve in the chiral time direction according to the Hamiltonian

$$
h_{1 \mathrm{~d}}^{R}(x)=\sum_{n}\left\{t_{n}(x) c_{n}^{R \dagger} c_{n+1}^{R}+t_{n-1}^{*}(x) c_{n}^{R \dagger} c_{n-1}^{R}\right\} .
$$

In this language the Green's function is given by

$$
G^{R}\left(n, x ; n^{\prime}, x^{\prime}\right)=<0\left|c_{n}^{R} P \exp \left(i \int_{x^{\prime}}^{x} d x_{1} h_{1 \mathrm{~d}}\left(x_{1}\right)\right) c_{n^{\prime}}^{R \dagger}\right| 0>
$$


for $x>x^{\prime}$. The symbol $P \exp ()$ denotes a time-ordered exponential. Note that there is no vacuum amplitude in the denominator because we are calculating the single particle green's function rather than the propagator for a single particle added to a filled fermi sea, which is the object usually studied in many-body physics [22]. The absence of a denominator in eq (4) is a crucial simplification that allows disorder averaging as discussed below.

The complex conjugate of the Green's function can be calculated from an expression analogous to eq (4) by introducing conjugate fermions, $c^{A \dagger}$ and $c^{A}$ which evolve according to a conjugate hamiltonian (obtained from eq (3) by making the replacements $c^{R} \rightarrow c^{A}, t \rightarrow-t^{*}$ and $\left.t^{*} \rightarrow-t\right)$.

Localization, or its absence, is established by calculation of the disorder-averaged diffuson propagator $\left|G_{E}^{R}\left(n, x ; n^{\prime}, x^{\prime}\right)\right|^{2}$. For this purpose it is neccessary to simultaneously introduce both sets of fermions evolving according to the total hamiltonian

$$
h_{1 \mathrm{~d}}(x)=h_{1 \mathrm{~d}}^{R}+h_{1 \mathrm{~d}}^{A}=\sum_{n}\left\{t_{n} A_{n}+t_{n}^{*} A_{n}^{\dagger}\right\} .
$$

Here $A_{n}=c_{n}^{R \dagger} c_{n+1}^{R}-c_{n+1}^{A \dagger} c_{n}^{A}$. The diffuson is then given by

$$
\begin{array}{r}
\left|G\left(n, x ; n^{\prime}, x^{\prime}\right)\right|^{2}= \\
<0\left|c_{n}^{A} c_{n}^{R} P \exp \left(i \int_{x^{\prime}}^{x} d x_{1} h_{1 \mathrm{~d}}\left(x_{1}\right)\right) c_{n^{\prime}}^{R \dagger} c_{n^{\prime}}^{A \dagger}\right| 0>.
\end{array}
$$

Eq (6) provides an exact formal expression for calculating the diffuson for a given disorder. The task now is to average over different realizations of the disorder potential which is taken to be gaussian white noise with correlations $\left[t_{n}^{*}(x) t_{m}\left(x^{\prime}\right)\right]_{\mathrm{imp}}=D \delta_{n, m} \delta\left(x-x^{\prime}\right)$ and $\left[t_{n}(x)\right]_{\mathrm{imp}}=\left[t_{n}(x) t_{m}\left(x^{\prime}\right)\right]_{\mathrm{imp}}=0$ 10 12], where [... $]_{\mathrm{imp}}$ denotes an average over disorder. Due to the absence of a denominator in eq (6) this average is easily performed. It is neccessary only to evaluate

$$
\left[P \exp \left(i \int_{x^{\prime}}^{x} d x_{1} h_{1 \mathrm{~d}}\left(x_{1}\right)\right)\right]_{\mathrm{imp}}=\exp \left\{-\bar{h}_{\text {int }}\left(x-x^{\prime}\right)\right\}
$$

where

$$
\bar{h}_{\mathrm{int}}=\frac{1}{2} D \sum_{n}\left(A_{n}^{\dagger} A_{n}+A_{n} A_{n}^{\dagger}\right) .
$$

Eq (8) can be verified by expanding the exponentials in eq (7). The rough content of eq (7-8) is that for calculating averages, the fermions may be taken to evolve according to an effective Hamiltonian $\bar{h}_{\text {int }}$ which is not random and does not depend on $x$. It is an interacting hamiltonian since $A_{n}$ is bilinear.

Consider a state in which a single site $n$ is simultaneously occupied by R and A fermions-below this state will be identified as a magnon localized at site $n$. The effect of $\bar{h}_{\text {int }}$ on such a state is to cause both fermions to hop together onto a neighbouring site. This physics can be brought out clearly by defining $J_{n}^{z} \equiv \frac{1}{2}\left(c_{n}^{R \dagger} c_{n}^{R}-c_{n}^{A} c_{n}^{A \dagger}\right)$, $J_{n}^{+} \equiv J_{n}^{x}+i J_{n}^{y} \equiv c_{n}^{R \dagger} c_{n}^{A \dagger}, J_{n}^{-} \equiv\left(J_{n}^{+}\right)^{\dagger}$, which satisfy the $\mathrm{su}(2)$ algebra, and $N_{n} \equiv c_{n}^{R \dagger} c_{n}^{R}+c_{n}^{A} c_{n}^{A \dagger}$, which commutes with all the $J$ 's. In terms of these operators

$$
\bar{h}_{\text {int }}=D \sum_{n}\left(N_{n}-\frac{1}{2} N_{n} N_{n+1}-2 \vec{J}_{n} \vec{J}_{n+1}\right)
$$

- evidently a Heisenberg ferromagnet. As usual the vacuum $\mid 0>$ is the ground state and exact low-lying excitations are magnons obtained by constructing plane-waves from the localized magnons mentioned above. Explicitly, a magnon of wave-vector $k$ is given by $\sum_{n} J_{n}^{+} \exp i k n \mid 0>$ and has eigenvalue $2 D(1-\cos k)$.

The exact diffuson propagator can now be straightforwardly calculated by substituting eq (7) in eq (6) and expanding $c_{n^{\prime}}^{R \dagger} c_{n^{\prime}}^{A \dagger} \mid 0>$ and $<0 \mid c_{n}^{A} c_{n}^{R}$ in terms of magnons. The result is

$$
\left[\left|G\left(n, x ; n^{\prime}, x^{\prime}\right)\right|^{2}\right]_{\mathrm{imp}}=\theta\left(x-x^{\prime}\right) \times
$$

$\int_{-\pi}^{+\pi} \frac{d k}{2 \pi} \exp i k\left(n-n^{\prime}\right) \exp \left\{-2 D(1-\cos k)\left(x-x^{\prime}\right)\right\}$

in agreement with eq (98) of Balents et al. 12. The physical content of eq (10) is that the electrons move ballistically in the chiral direction and diffuse in the transverse direction (diffusion constant $=D$ ); this is revealed, e.g., by using eq (10) to calculate the density response function [23].

Next consider the directed wave problem. The electron is assumed to be initially localized at the origin of the co-ordinate system $n^{\prime}=0, x^{\prime}=0$. After it moves ballistically in the chiral direction to a location $x$, the amplitude to be in layer $n$ is given by $G^{R}\left(n, x ; n^{\prime}=\right.$ $\left.0, x^{\prime}=0\right)$ and the position of the wave-packet centre $<n>=\sum_{n} n\left|G^{R}(n, x ; 0,0)\right|^{2}$. By symmetry, evidently $[\langle n\rangle]_{\mathrm{imp}}=0$; and the mean-square deflection of the wave-packet centre is therefore

$$
\left[<n>^{2}\right]_{\mathrm{imp}}=\sum_{n, m} n m\left[|G(n, x ; 0,0)|^{2}|G(m, x ; 0,0)|^{2}\right]_{\mathrm{imp}} .
$$

The large $x$ asymptotic behaviour of $\left[\langle n\rangle^{2}\right]_{\mathrm{imp}}$ is desired.

To perform this calculation it is neccessary to introduce two sets each of $R$ and $A$ fermions $\left(c^{R}, c^{A}, d^{R}, d^{A}\right)$ which evolve according to the hamiltonian of eq (5) but with $A_{n} \rightarrow c_{n}^{R \dagger} c_{n+1}^{R}-c_{n+1}^{A \dagger} c_{n}^{A}+(c \rightarrow d)$. Repeating the previous arguments,

$$
\begin{array}{r}
{\left[|G(n, x ; 0,0)|^{2}|G(m, x ; 0,0)|^{2}\right]_{\mathrm{imp}}=} \\
<0\left|c_{n}^{A} c_{n}^{R} d_{m}^{A} d_{m}^{R} \exp \left\{-\bar{h}_{\mathrm{int}} x\right\} d_{0}^{R \dagger} d_{0}^{A \dagger} c_{0}^{R \dagger} c_{0}^{A \dagger}\right| 0>.
\end{array}
$$


Here $\bar{h}_{\text {int }}$ is given by eq (8) but with $A_{n}$ redefined as above. The important states are (localized) two-magnon states which are of two kinds. In the first type a $c^{R}-c^{A}$ pair occupies one site while a $d^{R}-d^{A}$ pair occupies another; in the second type $c^{R}$ is paired with $d^{A}$ and $c^{A}$ with $d^{R}$ [24]. These states are closed under the action of $\bar{h}_{\text {int }}$ which generally causes paired fermions to hop together to a neighbouring site. An exception is when the two pairs occupy adjacent sites, in which case the fermions may switch partners and a state of one kind is transformed into the other. Thus the two-magnon states define a sort of two-body problem with a contact interaction of a kind familiar from ordinary ferromagnetism [17]. Following the standard method explicit forms for the two-magnon eigenstates are obtained which can be used to straightforwardly evaluate eq (11-12); details will be given elsewhere [21]. Contrary to the naive assumption that the centre undergoes a random walk, and hence $\left.[<n\rangle^{2}\right]_{\mathrm{imp}} \sim x$, exact calculation reveals that $\left[\langle n\rangle^{2}\right]_{\mathrm{imp}}=\sqrt{(2 D x) / \pi}$.

Finally consider multilayers that are of finite size in both chiral and transverse direction thus permitting electrons to wind around the sample in the chiral direction. During each circumnavigation, the electrons will typically diffuse a distance $\sqrt{D C}$ in the transverse direction. Consequently there are two distinct regimes depending on whether $N \ll \sqrt{D C}$ (zero-dimensional limit) or $N \gg \sqrt{D C}$ (one-dimensional limit). Here $N=$ number of layers in the multilayer. Finite sized samples are difficult to analyse due to the complex interference produced by winding paths. However, the conductance fluctuations can be calculated using diagrammatic perturbation theory within the approximation normally used for diffusive electrons [8]. The result is $\left[(\delta g)^{2}\right]_{i m p}=A\left(e^{2} / h\right)^{2}$ (one-dimensional limit) and $\left[(\delta g)^{2}\right]_{i m p}=A^{\prime}\left\{(C D) / N^{2}\right\}\left(e^{2} / h\right)^{2}$ (zero-dimensional limit). Here $A$ and $A^{\prime}$ are constants of order unity which we have not computed explicitly [25]. Conductance fluctuations are of order $e^{2} / h$ in the one-dimensional limit as they are for ordinary metallic grains. The larger non-universal result for the zero-dimensional limit may be interpreted as follows: The electron moves a distance $N^{2} / D \ll C$ in the chiral direction before it diffuses into the phase-randomizing probes. The sample therefore breaks up into $C D / N^{2}$ incoherent blocks, each with independent conductance fluctuations of order $e^{2} / h$. Although the perturbative results given here have an appealing physical interpretation, the validity of these results deserves further study via simulation or nonperturbative analysis (which may be possible in the zerodimensional limit 26]). By the familiar "ergodic hypothesis" of mesoscopic physics [8] we expect the statistical fluctuations of the conductance calculated here would be experimentally manifested as fluctuations in the conductance of a given specimen when a tunable parameter, e.g., the magnetic field, is varied.
It is interesting to compare the method of this paper with other field theory representations of the chiral model. Because single particle properties are being calculated, eq $(3-8)$ would remain valid even if the fermions were replaced by bosons $\left(c^{R}, c^{A} \rightarrow b^{R}, b^{A}\right)$ yielding an interacting boson representation of the chiral model rather than the Heisenberg ferromagnet analysed here. If fermions and a redundant set of bosons are introduced, the supersymmetric representation of Balents et al. 12] results. It is very convenient to be rid of the unneeded bosons, e.g., while analysing the directed wave problem; but it is important to emphasize that the bosons are optional only for the infinite system. For a finite circumference the boundary conditions on eq (1) change and it becomes neccessary to introduce bosons whether operator methods (this paper) or functional methods (Balents et al.) are used.

In summary, the main results of this paper are: a mapping of chiral waves onto a Heisenberg spin-chain with ferromagnetic coupling; calculation of the diffuson propagator, eq (10), which shows the surface of a quantum hall multi-layer to be an anisotropic metal in the infinite size limit; diagrammatic calculation of conductance fluctuations in two diffusive mesoscopic regimes for finite sized multilayers; and the exact result that the meansquare deflection of a directed wave grows as the square root of the distance it has propagated.

It is a pleasure to thank Leon Balents and Matthew Fisher for illuminating discussions and for patient explanation of the contents of ref 10,12] and Yi Kuo Yu for discussion of conductance fluctuations. Thanks are also due to Matthew Fisher for hospitality at the ITP Santa Barbara where this work was initiated. This work was supported by NSF Grant PHY 94-07194, startup funds from CWRU and an Alfred P Sloan Research fellowship.

[1] P.A. Lee and T.V. Ramakrishnan, Rev Mod Phys 57, 287 (1985).

[2] D. Belitz and T.R. Kirkpatrick, Rev Mod Phys 66, 261 (1994)

[3] B. Huckestein, Rev Mod Phys 67, 357 (1995).

[4] B.I. Halperin, Phys Rev B25, 2185 (1982).

[5] For recent experiments, see L. Balicas et al., Phys Rev Lett 75, 2000 (1995); S.M. McKernan et al., Phys Rev Lett 75, 1630 (1995); and references therein.

[6] H.L. Stormer et al., Phys Rev Lett 56, 85 (1986).

[7] J.T. Chalker and P.D. Coddington, J Phys C 21, 2665 (1988).

[8] P.A. Lee, A.D. Stone and H. Fukuyama, Phys Rev B35 1039 (1987).

[9] J.T. Chalker and A. Dohnen, Phys Rev Lett 75, 4496 (1995). 
[10] L. Balents and M.P.A. Fisher, Phys Rev Lett 76, 2782 (1996).

[11] Y. Kim, preprint (cond-mat 9601050).

[12] L. Balents, M.P.A. Fisher and M.R. Zirnbauer, preprint (cond-mat 9608049).

[13] K.B. Efetov, Adv Phys 32, 53 (1983).

[14] L. Golubovic, S. Feng and F. Zeng, Phys Rev Lett 67, 2115 (1991).

[15] L. Saul, M. Kardar and N. Read, Phys Rev A 45, 8859 (1992).

[16] A.A. Ovichinnikov and N.S. Erikhman, Zh Eksp Teor Fiz 67, 1474 (1974); A. Madhukar and W. Post, Phys Rev Lett 39, 1424 (1979).

[17] H.A. Bethe, Z. Physik 71, 205 (1931). For a textbook account see R.P. Feynman, Statistical Mechanics, Ch 7 (Addison-Wesley, 1972).

[18] J.P. Bouchaud, D. Touati, and D. Sornette, Phys Rev Lett 68, 1787 (1992).

[19] S. Feng, L. Golubovic and Y.-C. Zhang, Phys Rev Lett 65, 1028 (1990).

[20] R. Friedberg and Y.K. Yu, Phys Rev E49, 5755 (1994).

[21] H. Mathur, unpublished.

[22] A.L. Fetter and J.D. Walecka, Quantum Theory of Many Particle Systems (McGraw Hill, New York, 1971).

[23] D. Forster, Hydrodynamics, Broken Symmetry and Correlation Functions (WA Benjamin, 1975).
[24] The states for which a single site is occupied by all four fermions are special and may be considered to be of either type.

[25] To be precise, we have explicitly evaluated the diagram in fig 5 (a) of ref [8] which yields $A=4 \zeta(4) / \pi^{4}$ and $A^{\prime}=2 \zeta(2) / \pi^{2}$. Diagrams 5 (b-e) should yield the same parametric dependence but the coefficients have not been computed.

[26] Y.K. Yu, private communication.

Fig 1. A quantum hall multilayer. 